\title{
Versatilidad de un Servicio de Cardiología y Cirugía Cardiovascular en tiempos de COVID 19
}

\author{
Manuel Quiroz ${ }^{1,2}$, Javier Labbé $1,2,3$. \\ 1. Cirujano Cardiovascular, Unidad de Cardiología y Cirugía Cardiovascular, Hospital Clínico Regional de Antofagasta, \\ Dr. Leonardo Guzmán, Antofagasta, Chile. \\ 2. Departamento de Ciencias médicas, Facultad de Medicina y Odontología, Universidad de Antofagasta, Antofagasta, Chile. \\ 3. Jefe Unidad de Cardiología y Cirugía Cardiovascular, Hospital Clínico Regional de Antofagasta, \\ Dr. Leonardo Guzmán, Antofagasta, Chile.
}

\section{Sr. Editor:}

Antofagasta es una ciudad del norte de Chile ubicado a $1400 \mathrm{~km}$ de la capital, en un país altamente centralizado con un número de especialistas por millón de habitantes bajo la media nacional. ${ }^{1-3}$

El servicio de Cardiología y Cirugía Cardiovascular presta atención a 1,4 millones de habitantes desde Arica a Copiapó.

El funcionamiento normal de nuestro servicio se inicia cuando un paciente que ingresa al servicio de urgencias por un cuadro clínico de probable etiología cardiovascular (como el síndrome coronario agudo) es evaluado por los residentes de cardiología (quienes hacen turnos de 24 hrs en la unidad de 36 camas). Si el paciente está grave y tiene compromiso hemodinámico se ingresa a la Unidad de Cuidados Intensivos Cardiovasculares que tiene una capacidad de 10 pacientes, residentes de turno 24 horas y 2 médicos diurnos. Cuenta con aislamientos, proporción de 1 enfermera de experiencia por cada 3 pacientes y unidad de rehabilitación cardiovascular en fase 1 de cuidados intensivos. En el caso favorable de un paciente estable se hospitaliza en la unidad de Camas Medias con capacidad de 34 pacientes y 2 aislamientos. En la misma evaluación se determina si requiere estudio de urgencia en el laboratorio de hemodinamia y, además, si requiere algún tipo de asistencia como balón de contrapulsación intra aórtico o 
sistema de oxigenación de membrana extracorpórea (ECMO).

Si el paciente cumple criterios de revascularización percutánea se realiza el procedimiento y se traslada a UCI cardiovascular para observación.

Si requiere cirugía, muy probablemente se operaría antes de una semana y la conducta quirúrgica habitual del servicio es cirugía sin circulación extracorpórea y dependiendo de la anatomía, revascularización con arteria mamaria interna izquierda y derecha anastomosada en Y (BIMA) más algún puente venoso. Sin embargo, el análisis y el tratamiento se discuten caso a caso, con el objetivo de ofrecer al paciente la alternativa que más lo favorece.

El paciente sale de pabellón a la UCI con protocolo "fast track" de extubación a las 4 horas, y comienza la rehabilitación cardiovascular en fase 1 , y al $4^{\circ}$ día pasa a fase 2 en el gimnasio de la unidad que cuenta con bicicletas de pies y manos, ergo espirometría, etc.

Al momento del alta, generalmente al $5^{\circ}$ o $6^{\mathrm{O}}$ día el paciente egresa del hospital con hora asignada para control de cirugía en 7 días, curación por la enfermera de rehabilitación y control por cardiólogo al mes.

Se clasifica según clínica y fracción de eyección para determinar el tiempo de rehabilitación presencial a indicar.

\section{Problemática COVID-19 en Antofagasta}

Nuestra región sufre especialmente por esta pandemia. No se practicó pesquisa precoz ni contención en Atención Primaria de Salud (APS). Abundan los campamentos de inmigrantes y personas en situación de vulnerabilidad que viven en los cerros, tal como el Sacerdote Felipe Berríos quien, emblemáticamente, vive en uno de estos campamentos de forma similar a lo que hacía en su residencia anterior en África. Nuestra región posee el segundo lugar en cantidad de hogares en campamentos (7641), solo superada por la región de Valparaíso. 4

En la región de Antofagasta al 12 de Julio hay 12601 casos confirmados y 259 fallecidos, con 2023 casos activos. La mayor mortalidad la tiene Calama, con 149 fallecidos y una tasa de mortalidad de 78\%, mientras Antofagasta está en segundo lugar con 86 fallecidos y una tasa de mortalidad de $20 \% .^{5}$ La situación de Calama, cuyo funcionamiento hospitalario habitual consideraba a Antofagasta como su principal refuerzo en la resolución de casos complejos, se vio afectada por el escenario actual, en el cual de un día para otro no pudieron contar con apoyo.

Existe pesquisa insuficiente y retraso en la entrega de resultados (hasta diez días); hubo alrededor de 3000 muestras sin procesar en un plazo mayor a lo recomendado.

\section{Alternativas frente a la pandemia}

Frente a este escenario completamente nuevo y fuera de la escala habitual, nuestras alternativas como servicio de cardiología y cirugía cardiovascular eran: dejar de lado a nuestros pacientes cardiovasculares de la Macro Zona Norte y abocar nuestra estructura al manejo de pacientes COVID 19, o resguardarnos para evitar convertirnos en contagios (medidas tomadas por otros servicios clínicos (que prácticamente cerraron la atención) o hacernos cargo del problema buscando alternativas para mantener continuidad 
de la atención cardiovascular del norte de Chile.

Se definió una estrategia que fue la reducción de la actividad, dividir los equipos y turnar el trabajo presencial cada siete días en las unidades críticas de cardiología, pasar de turnos de 12 horas a 24 horas para minimizar las posibilidades de contagio y exposición de más de un turno.

Se destinó tiempo a educación de Infecciones Asociadas a Atención de Salud (IAAS) a todo el personal en forma reiterada y se generaron capacitaciones vía E-learning mediante el apoyo de diferentes plataformas digitales afines a este objetivo, las cuales tuvieron una alta convocatoria e impacto en los resultados clínicos.

\section{Contribución a la necesidad de camas críticas en modalidad de UCI extendida}

El problema se abordó generando una UCI general en la Unidad de Recuperación de Anestesia, que contó inicialmente con 8 camas y luego se extendió a 15. El objetivo era ser una UCI general para pacientes COVID 19 (-), contribuir a liberar camas para pacientes COVID 19 (+) graves y dar un manejo adecuado a los enfermos de las patologías que, lamentablemente, en todo nuestro territorio no dejaron de ser prevalentes (Enfermedad cerebro vascular, trauma craneoencefálico grave, paciente crítico quirúrgico, patología oncológica de resolución quirúrgica entre otros).

Los pacientes críticos cardiovasculares se manejaban de forma habitual en la UCI cardiovascular. Sin embargo, con el avance de la pandemia transformamos la UCI coronaria que cuenta con 10 camas, a una unidad de aislamiento en UCI COVID (+). En este escenario decidimos realizar su hospitalización en la nueva UCI de recuperación de anestesia, por lo que tuvimos que reinventar esta unidad para asumir el desafío de recibir pacientes cardio quirúrgicos y cardiológicos graves.

La UCI de recuperación de anestesia se dotó mediante la conversión del personal de enfermería de camas medias, quienes se entrenaron junto a colegas de mayor experiencia para funcionar como enfermeras y enfermeros de cuidados críticos, nuestro equipo de kinesiólogos rehabilitadores de fase 2, se convirtieron en kinesiólogos de UCI general y los médicos pasaron de anestesistas cardiovasculares, cirujanos cardiovasculares y cardiólogos a residentes de la UCI general de recuperación de anestesia.

\section{Transformación de la actividad presencial}

Este esfuerzo realizado por todos los estamentos de la unidad, sumado a las restricciones para evitar contagios, causó que los policlínicos fueran suspendidos. Sin embargo, se decidió mantener el control de los pacientes por vía telefónica, para pesquisar pacientes con necesidad de atención oportuna. La rehabilitación cardiovascular presencial se convirtió en tele rehabilitación mediante plataformas de audio y video, canales de información como aplicaciones de mensajería telefónica, mediante la creación de grupos altamente participativos. Las curaciones volvieron a los enfermeros de pabellón y los controles presenciales de cirujanos se redujeron a los casos más complejos.

El compromiso de la unidad y el trabajo multidisciplinario han permitido tener buenos resultados, tanto en la UCI extendida, como en el cuidado de los pacientes cardiovasculares graves. El control telefónico ha permitido dar solución a pacientes con agotamiento de generador de marcapasos y arritmias, entre otros, y la posibilidad de contar 
con camas COVID 19 (-) ha facilitado mantener el funcionamiento del servicio de hemodinamia de urgencia sin contratiempos.

Respecto a nuestra conducta quirúrgica se ha adaptado a lo recomendado en situaciones de pandemia, privilegiando resolver lo más grave y considerando en cada caso la posibilidad de una resolución mediante manejo híbrido si el paciente es candidato quirúrgico. 6

Hemos logrado dar resolución a 45 casos cardio quirúrgicos, entre los que contamos dos disecciones aórticas tipo A, 80 casos de revascularización percutánea, 30 casos de marcapasos de urgencia y 2 estudios electrofisiológicos y ablaciones entre otros; como UCI en pabellón de recuperación hemos atendido 84 pacientes hasta la fecha.

Con esto quisiéramos resaltar una decisión de servicio frente a este escenario, en el cual decidimos hacernos parte del combate de la pandemia dando atención a los pacientes COVID 19 (+) en la UCI cardiovascular y a los pacientes postergados de patología general mediante una estrategia que aprovechó al máximo la versatilidad de las especialidades que trabajan en nuestro servicio y con esto contribuimos a disminuir la mortalidad de los enfermos COVID 19 (-), que no serían contados en los reportes diarios.

Agradecemos a todo el personal del servicio de cardiología y cirugía cardiovascular por la enorme entrega y el trabajo realizado por los enfermos del norte de Chile.

\section{Referencias}

1.- GUILlOU M, CARABANTES J, BUSTOS V. Disponibilidad de médicos y especialistas en Chile, Rev Med Chile 2011; 139: 559-70.

2.- GUILlOU M, Estudio de Brechas de Demanda y Oferta de Médicos Especialistas en Chile. Informe Final. Banco Mundial. Gobierno de Chile-Ministerio de Salud. Departamento de Estudios de Recursos Humanos. Subsecretaria de Redes Asistenciales. Ministerio de Salud. Enero de 2010.

3.- INFORME DE BRECHAS DE PERSONAL DE SALUD POR SERVICIO DE SALUD. Glosa 01, letra i de la ley de Presupuestos. Año 2016. Departamento de Planificación y Control de Gestión de Recursos Humanos. Marzo 2016. http://web.minsal.cl/wp-content/uploads/2015/08/Informe-Brechas-RHS-en-SectorP\%C3\%BAblico_Marzo2016.pdf

4.- CATASTRO NACIONAL DE CAMPAMENTOS 2019. MINVU, 2019. https://storymaps.arcgis. com/stories/dfe1fe1afd334ec790f879e736a5af5e

5.- INFORME EPIDEMIOLÓGICO No 33, Enfermedad por SARS-CoV-2 (COVID - 19), Chile, 13 de Julio 2020, Departamento de Epidemiología, Ministerio de Salud.https://www.minsal.cl/ wp-content/uploads/2020/07/InformeEPI130720.pdf

6.- ENGELMAN D, LOTHER S, GEORGE I, FUNK D J, AILAWADI G,ATLURI P, GRANT M C,HAFT J W, HASSAN A, LEGARE JF ,. WHITMAN, G J R, ARORA R C. Adult cardiac surgery and the COVID-19 pandemic: Aggressive infection mitigation strategies are necessary in the operating room and surgical recovery. J. Thorac. Cardiovasc. Surg. 2020; 160: 447-51. 